\title{
Global Mixing Indices for Single Screw Extrusion
}

\author{
N. Domingues, A. Gaspar-Cunha, J. A. Covas \\ Department of Polymer Engineering, University of Minho, 4800-058 Guimarães, Portugal \\ URL: http://ipc.uminho.pt \\ e-mail: (nat-domingues, agc, jcovas)@dep.uminho.pt
}

ABSTRACT: The present work presents numerical simulations of dispersive and distributive mixing of a two phase system being processed in a single screw extruder under various conditions. Models were developed to quantify the degree of mixing of liquid-liquid and solids-liquid systems, for a given set of material properties, operating conditions and extruder geometry.

Key words: Mixing, Extrusion, Distribution, Dispersion.

\section{INTRODUCTION}

Single screw extruders are a key component of extrusion lines for the production of plastics products, being used with the aim of pumping a homogeneous melt at the highest possible rate, i.e., they must generate a well distributed and well dispersed melt containing liquid and/or solid additives. Although discriminating between the mixing efficiencies of different screws is not a simple task, this is a relevant practical problem, as the choice of an adequate screw, together with the selection of appropriate operational conditions, may induce the required mixing intensity levels [1]. This work proposes the use of global quantitative dispersive and distributive mixing indices to be used for that purpose.

The indices are computed from the predicted final size/shape of liquid droplets or solid particles that are assumed to exist as suspended units in the melt.

Such computations require the coupling of the modelling of flow along the screw, with the description of the evolution of morphology. In the case of liquid-liquid systems, distributive mixing depends essentially on droplet stretching and residence time, while dispersion depends on drop break-up and coalescence. Distributive mixing of solid-liquid systems depends on residence time and particles location (calculated via entropy), while dispersive mixing considers rupture and erosion phenomena [2].

\section{MIXING QUANTIFICATION}

Mixing involves the distribution and dispersion of the minor component in the polymeric matrix. Both phenomena are promoted simultaneously or sequentially via the shear rate applied. Different systems are considered here: a) liquid-liquid system (represented by two immiscible polymers or a melted polymer with a liquid additive); b) solidliquid system (comprising a melted polymer with solid additives). Different mathematical models were developed for each system.

\subsection{Liquid-liquid systems}

Mixing two immiscible polymers generates a two phase system, where small drops of the minor component are suspended in the major component, the polymeric matrix. These drops can suffer breakup (breaking into two smaller drops) or coalescence (two drops joining together), which depends on various parameters, such as relative viscosity, capillary number and residence time [3].

Dispersive mixing is estimated by computing the change in drop diameter $(d)$ relative to the original value $\left(d_{i}\right)$, at any location. A global mixing index is computed considering an average of $N$ drops through the following equation [2]:

$$
\operatorname{mix}_{\text {disp }}=\frac{\sum_{j}^{N}\left[\left(\frac{d}{d_{i}}\right)_{j} \times\left(1-\frac{d}{d_{i}}\right)_{j}\right]}{\sum_{j}^{N}\left(\frac{d}{d_{i}}\right)_{j}}
$$

Distributive mixing is quantified using the concept of affine deformation of a drop, which depends on shear rate and residence time [3]. The mixing index used depends on the actual width of the drop $(B)$ and on the original value of drop diameter $\left(d_{i}\right)[2]$ : 


$$
\operatorname{mix}_{d i s t}=\frac{\sum_{j}^{N}\left[\left(\frac{d}{d_{i}}\right)_{j} \times\left(1-\frac{B}{d_{i}}\right)_{j}\right]}{\sum_{j}^{N}\left(\frac{d}{d_{i}}\right)_{j}}
$$

Both mixing indices are dimensionless and range in the interval $[0,1]$. Values close to zero correspond to poor mixing.

\subsection{Solids-liquid Systems}

Solid additives are generally agglomerates comprising indivisible particles clustered together. Agglomerates can rupture (break-up into two smaller agglomerates) or erode (detachment of indivisible particles from the surface of the agglomerate), depending on the flow hydrodynamic forces and cohesive forces of the agglomerate [4]. A Monte Carlo method is used to compute the probability of break-up combined with a rupture/erosion model [5]. To quantify the dispersion in the system, equation (1) is used, where $d$ and $d_{i}$ are replaced by the agglomerate diameter and the initial value of the agglomerate diameter, respectively, and $N$ is the total number of agglomerates.

The level of distributive mixing is assessed using the Shannon entropy $(S)$ :

$$
S=-\sum_{j=1}^{M} p_{j} \log p_{j}
$$

where $p_{j}$ is the probability of finding a particle in the bin $j$ and $M$ is the total number of bins in which the screw channel section is divided $[6,7]$.

The maximum value of the Shannon entropy is obtained when the probability of finding a particle is the same on each bin, i.e., $p_{j}=1 / M$. Dividing $S$ by $\log (M)$, a normalized Shannon entropy can be defined and used as mixing index ranging in the interval $[0,1]$.

\section{CASE STUDIES}

A prototype modular single screw extruder with L/D ratio of 30 and a barrel diameter (D) of $30 \mathrm{~mm}$ was used. The channel depth $(\mathrm{H})$ in the metering zone has $2 \mathrm{~mm}$, while in the feed section three different values were used, 3,5 or $7 \mathrm{~mm}$ (compression ratios CR- of 1.5, 2.5 and 3.5 respectively), as shown in figure 1 . The axial length of feed, compression and metering sections $\left(\mathrm{L}_{1}, \mathrm{~L}_{2}\right.$ and $\left.\mathrm{L}_{3}\right)$ are the same and equal to $300 \mathrm{~mm}$.

The properties of the HDPE T-100, Repsol YPF were used in the numerical simulation. In the case of the liquid-liquid system 10000 virtual drops were uniformly distributed in the matrix. The viscosity ration is 1 and the surface tension taken as $0.09 \mathrm{~N} / \mathrm{m}$. For the solid-liquid system, 10000 agglomerates were uniformly distributed in the matrix, each one comprising 100 indivisible particles, with a cohesive force of $1000 \mathrm{~Pa}$.

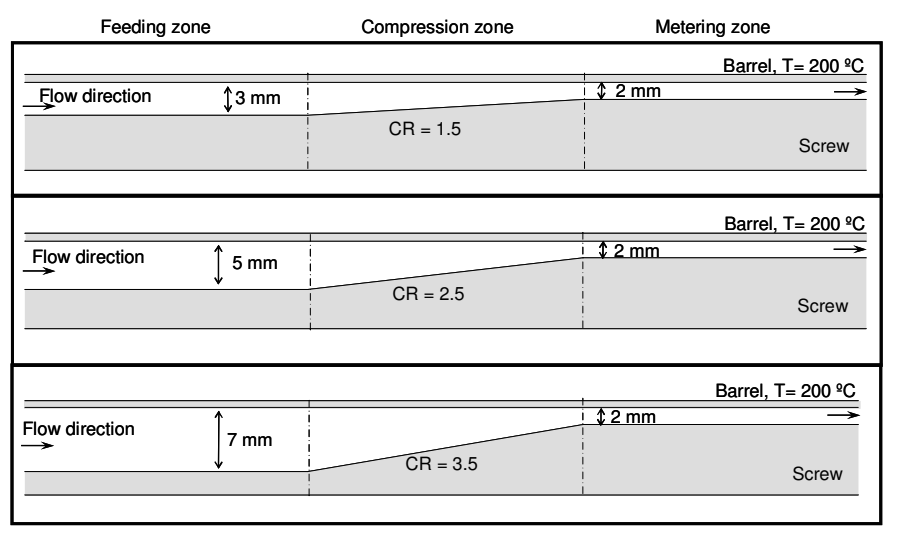

Fig. 1. Screw geometries with different compression ratio.

\section{RESULTS AND DISCUSSION}

\subsection{Influence of screw speed}

As shown in figure 2, the mixing degree (dispersive and distributive) of liquid-liquid systems decreases with the increase of screw speed. In spite of the increase of average shear rate with screw speed, the residence time decreases for higher screw speeds (this is shown in Figure 3). The balance between these two parameters determines the behaviour depicted in Figure 2.

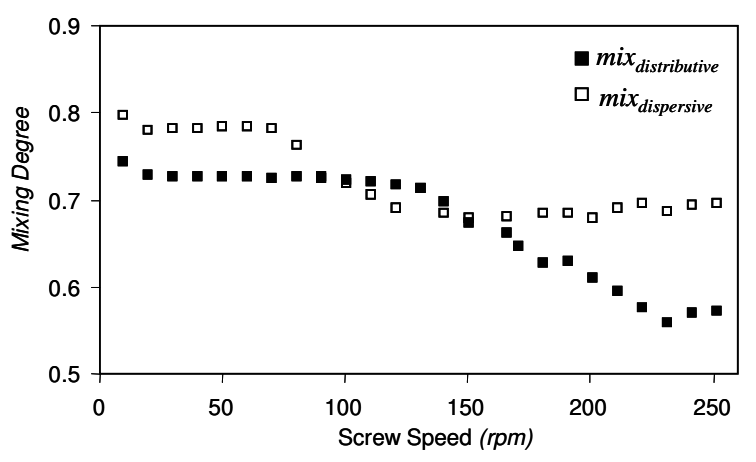

Fig. 2. Mixing indices for liquid-liquid systems. 


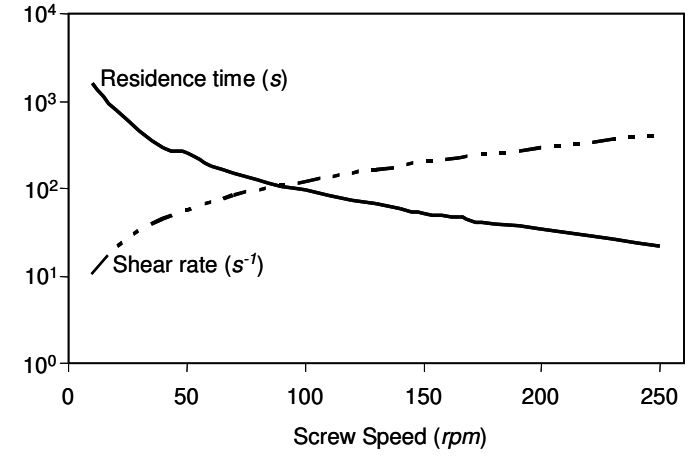

Fig. 3. Average of shear rate and particles residence time.

For solids-liquid systems (Figure 4), the effect of shear rate is noted when screw speed ranges between 10 and $60 \mathrm{rpm}$, while above $60 \mathrm{rpm}$ the particles residence time becomes too short to achieve a good dispersive mixing. Distributive mixing, as quantified by Shannon entropy, remains stable, since the particles were uniformly distributed in the beginning of the screw channel.

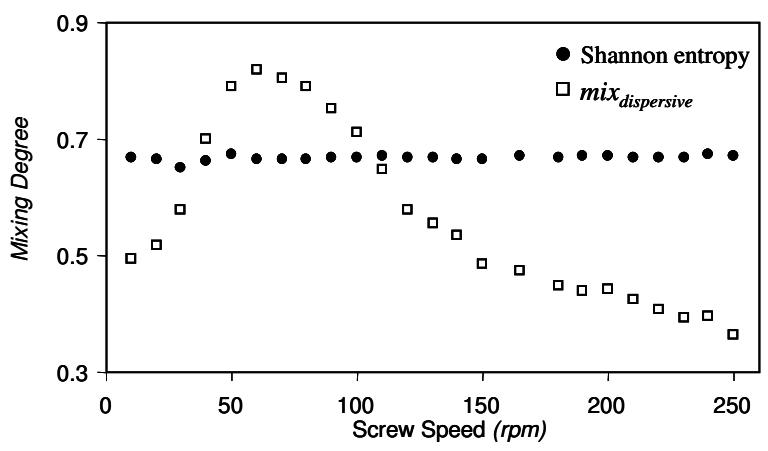

Fig. 4. Mixing indices for solids-liquid systems.

\subsection{Influence of screw geometry}

Changing the screw compression ratio produces the effect on the mixing indices presented in Figures 5 and 6. Somewhat unexpectedly, for equivalent screw speeds, the screw with lower compression ratio induces better liquid-liquid mixing at the low to medium screw speed range, the more compressive screw being more efficient only for high speeds (however, one should notice that changes in compression ratio were achieved by varying the channel depth of the feeding zone - Figure 1). Figure 7 shows that these differences in mixing are not explained by relative values of total residence time and average shear rate for each screw. However, if average shear stresses (average shear rate $*$ viscosity) and total deformation (average shear rate $*$ residence time) along the channel are computed (Figure 8), one may conclude that (with the exception of very low speeds) both are higher for the low compression screw up to $170-180 \mathrm{rpm}$, and for the higher compression screw for speeds above. In the case of solids-liquid mixing, two regimes can be detected (Figure 6), as already reported in Figure 4. More interestingly, the high compression screw is more efficient for the lower screw speed range, while the lower compression screw has more dispersive capacity at higher screw speeds. These results can be explained by the differences in average shear rate presented in Figure 7 (the change in behaviour occurring exactly at the same screw speed).
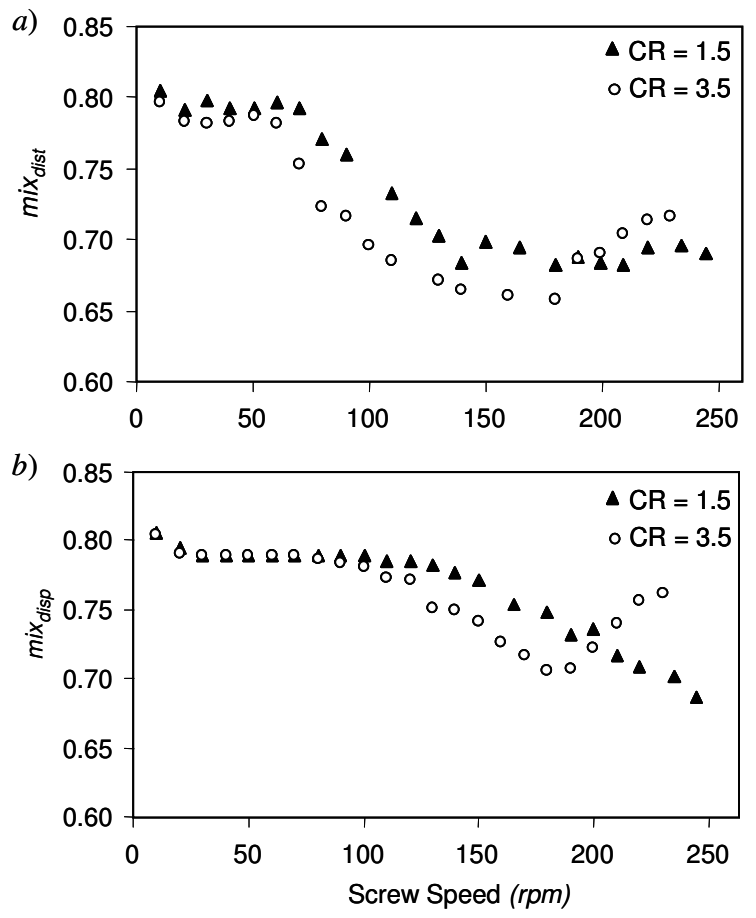

Fig. 5. Distributive and dispersive mixing indices on liquidliquid system

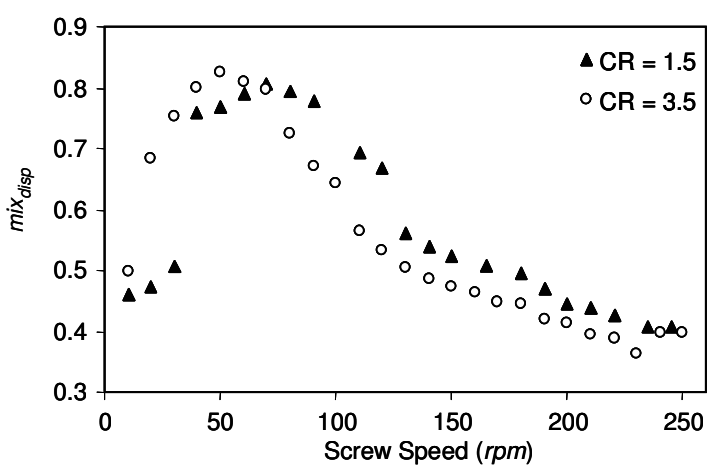

Fig. 6. Dispersive mixing index for solids-liquid system. 


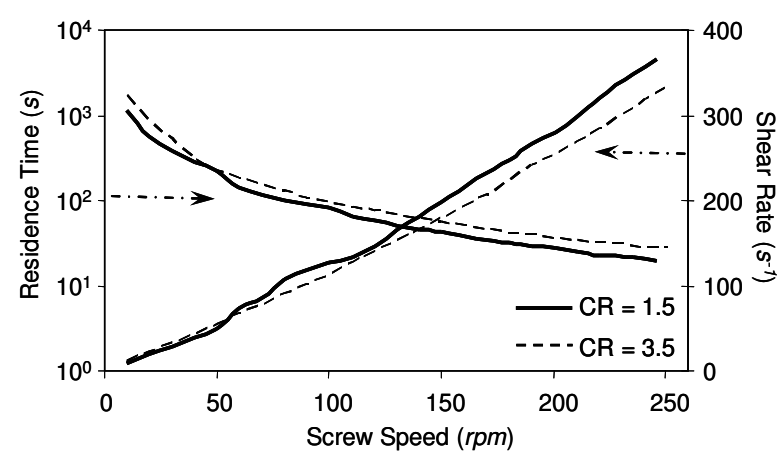

Fig. 7. Average shear rate and particles residence time.

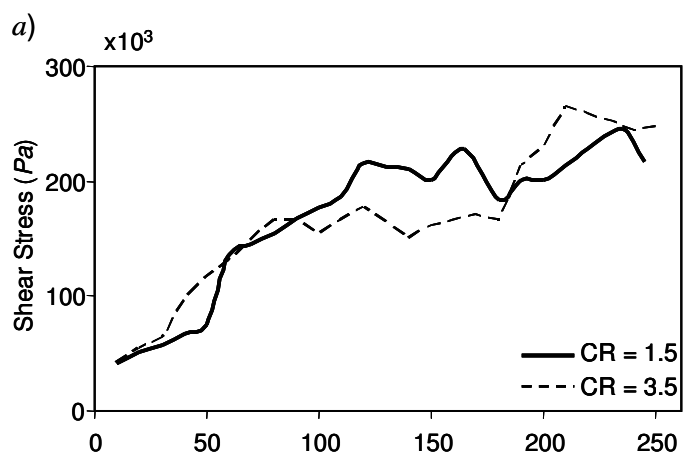

b)

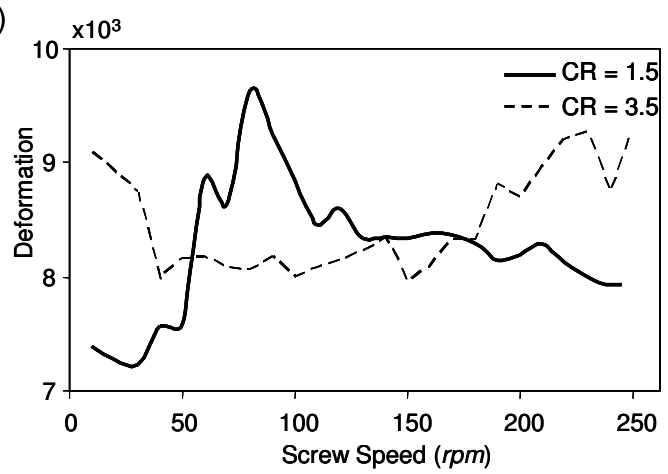

Fig. 8. Shear stress induced by flow and deformation.

\section{CONCLUSIONS}

Data from predictions of morphology evolution along the screw was used to generate mixing indices. These indices, ranging in the interval $[0,1]$, were used to compare the mixing efficiency of different screw geometries.

Mixing efficiency depends on material properties, operating conditions and screw geometry, this work showing that the methodology proposed is sensitive to the geometrical parameters.

\section{ACKNOWLEDGEMENTS}

Natália Domingues acknowledges FCT Portuguese Foundation (Fundação para a Ciência e Tecnologia) for grant SFRH/BD/19605/2004.

\section{REFERENCES}

1. Rauwendaal, Mixing in Polymer Processing, Marcel Deckker, Inc., New York (1991).

2. N. Domingues, A. Gaspar-Cunha, J.A. Covas, Predicting Distributive and Dispersive Mixing in Polymer Extrusion, In: Proc. NUMIFORM'07, Materials Processing and Design: Modeling, Simulation and Applications, eds, American Institute of Physics (2007) 1531-1536.

3. H.E.H Meijer, J.M.H. Janssen, Mixing of Immiscible Liquids, in Mixing and Compounding of Polymers, In: Mixing and Compounding of Polymers, eds, I. ManasZloczower, Z. Tadmor, Hanser Publishers, Germany, (1994) 85-147.

4. K. Alemaskin, I. Manas-Zloczower, M. Kaufman, 'Simultaneous Characterization of Dispersive and Distributive Mixing in a Single Screw Extruder', Proceedings of the Int. Conf. ANTEC 2003, Nashville, (2003).

5. N. Domingues, M. Camesasca, M. Kaufman, I. ManasZloczower, A. Gaspar-Cunha and J. A. Covas, 'Modelling Agglomerate Dispersion in Single Screw Extruders', Proceedings of the Int. Conf. ANTEC 2006 Charlotte, (2006).

6. C. E. Shannon, W. Weaver, The Mathematical Theory of Communication, Urbana, University of Illinois Press, (1948).

7. A. I. Khinchin, Mathematical Foundations of Information Theory, New York, Dover, (1957).

8. M. Camesasca, M. Kaufman, and I. Manas-zloczower, 'Quantifying fluid mixing with the Shannon entropy', Macromolecul. Theory Simul., 15,n 8, (2006) 595-607.

9. N. Domingues, A. Gaspar-Cunha, J.A. Covas, Computational and Experimental Study of Mixing in a Single Screw Extruder, In: Proc. 10th ESAFORM, Conference on Material Forming, eds, American Institute of Physics, (2007) 867-872. 